\section{A problematic} Israeli High Court dismissal of a

\section{torture complaint.} A commentary by Hans Draminsky Petersen, MD*

In her description of the case of Firas Tbeish (FT), Efrat Shir highlights some crucial weaknesses in the protection of detainees against torture and ill-treatment, inter alia, that the court does not deal, neither with ill-treatment, nor with psychological aspects of torture and ill-treatment; and proper medical documentation does not exist. A key issue is that Israeli authorities do not use the UN Istanbul Protocol (IP) for documentation of torture and ill-treatment and results of such examinations produced by external and independent experts are rejected.

FT was convicted of terrorist acts and was given a relatively mild sentence after a plea agreement. The court held that "particularly in view of the exceptional interrogation that the defendant experienced" the arrangement was found reasonable (para 13). ${ }^{1}$

On April 2, 2013, FT filed a complaint through his attorneys requesting a criminal

"para" refers to the respective paragraphs in the ruling.

*) Ex-member and vice-chair of the UN Subcommittee on Prevention of Torture (SPT) Correspondence to: hdp@dadlnet.dk investigation against his interrogators due to "a brutal course of psychological and physical torture" (para 14). He further requested the investigation of the members of the medical staff that allegedly were physically present in the interrogation room in order to provide medical treatment, but who did nothing to "stop the torture" (para 14).

The court ruled on November 26, 2018 that FT was not tortured. The following reflections on several aspects in this ruling develop further the arguments of Shir. There are two main challenges: How does Israel in practice define torture and how should torture be documented and appraised?

\section{Medical evidence produced during the period of interrogations}

In the period when "special means" were employed, FT was examined four times by a prison service physician; three of the examinations were done in the interrogation room and the medical notes are quoted as follows in the ruling (para 6).

1. September 19, 2012 the medical examination found "pain and swelling in the upper right molar area" and noted "Buccal swelling. Pain upon palpation. Periodontal abscess".

2. On Sept. 21, 2012 at 5:37 AM [the physician] found "his general condition is reasonable", his skin is "pale", and he suffers from diarrhea.

3. The same day at 6:03 AM, he was examined following complaints of pain in his knees, and it was noted that "in the examination - he appeared agitated. Red eyes. Did not sleep tonightinterrogation". The examination did not find reason for new treatment.

4. The same day at 6:42 PM, the petitioner was examined again, this time for complaints of pain in his left knee. 
The examination found "his general condition is reasonable", and he was given medication for swelling, pain and restricted movement of his knee.

The role of the doctor in a detention facility is to safeguard the detainee's health and to identify, document and report torture and ill-treatment (UN Mandela Rules). The reproduced documents are grossly insufficient in both respects. For example, none of them indicate the reason for the examination and where it took place, nor contain any history of symptoms and signs.

In the context of detention and application of "special means" the doctor should have in mind the possibility that the swelling of the mouth and the pathology of the knee could have had a traumatic cause. If swellings of the mouth on both the jaw ("periodontal") and the cheek ("buccal") arise acutely and are thought to be of infectious origin ("abscess") there is ground for further examinations, primarily by a dentist who has access to $\mathrm{X}$-ray examinations, or at least measurement of temperature and administration of penicillin. A description of the general health status of the teeth would have been useful to substantiate the likelihood of the diagnosis "abscess" /infection. On the other hand negative findings such as absence of hematomas are also highly relevant in the context of interrogations using "special means" where application of physical violence may take place or may be alleged. "Diarrhea" is not described (onset, appearance, frequency and concomitant pain vomiting and fever) and an examination of the abdomen is absent. "General condition" is not explained (e.g, blood pressure, pulse rate and temperature). Pain swelling and restricted movement of a knee are described perfunctorily and no diagnoses is suggested, notwithstanding that the most likely cause in a healthy young man is trauma.

The insufficiency of the medical notes concerning findings in the mouth and the absence of actions taken by the doctor could make the reader think that an obviously possible diagnosis of a traumatic lesion was disregarded in favour of the-for the authorities - more acceptable diagnosis of infection (given implicitly).

To fulfill the demands of the Mandela Rules the doctor should - in accordance with the IP-keep meticulous record on the relevant A) history, which should include physical and psychological traumas, B) the onset, nature, duration and gravity of symptoms and the $\mathrm{C}$ ) results /findings of an objective examination. When assessing the veracity of allegations of torture and illtreatment those three elements should be considered together appraising whether the history is consistent with the other elements, which constitutes D) a conclusion. Likely differential diagnosis should be mentioned and further actions (e.g. specialist examinations or treatment) may be required.

The quoted medical records fail in all of these: $\mathrm{A}$ and $\mathrm{D}$ are absent and $\mathrm{B}$ and $\mathrm{C}$ are insufficient.

It is remarkable that the Inspector of Interrogees' Complaints from the Department of Complaints against the ISA (in the following: "the Inspector") seems to rely on the prison medical service's description of the deceased medical doctor who carried out the four examinations of FT as "very meticulous" without assessing for him or herself the quality of the documents. The allegations of loss of consciousness during interrogations were rejected based on the fact that they were not mentioned in the medical records.

The inspector disregarded the knee and mouth pathologies described by the 
prison doctor, and FT's allegation that he lost a tooth as a consequence of torture is not commented. It is stated that "it would be expected that if there were substance to the claims, the medical records would show objective findings" (para 19). By disregarding documented lesions, it is implicitly concluded that there was no substance in FT's allegations. Moreover, the inspector disregarded the common knowledge that absence of marks does not prove that torture was not committed as underlined repeatedly in the IP.

The inspector's interviews with prison staff, including the medical service took place up until $3 \frac{1}{2}$ years after the event.

FT described that at some point he had a black spot in the eye that was hit during interrogation. He further alleged that he was shaken. An examination by an ophthalmologist with a CT scan could have revealed or ruled out a whip-lash maculopathy, which causes disturbances of the vision lasting for weeks or months. Neither these alleged abuses nor the symptoms were mentioned in the doctor's notes quoted in the ruling. The reader of the ruling could think that this reflects that either the doctor disregarded symptoms described by the detainee or that he was far from being proactive in his gathering of information from the detainee-an impression that is reinforced by the general extreme brevity of the notes.

\section{Medical complicity in ill-treatment and torture}

Whether or not the doctor was present during the interrogations cannot be deduced from the ruling, but notably three of the examinations were carried out in the interrogation room "although there was no particular medical urgency" (para 19). FT requested an investigation of the medical staff (para 19). The investigator's remarks mentioned in the ruling cannot constitute such an investigation.

The doctor's possible presence for the "special means" raises serious concerns about medical complicity in torture /illtreatment. The relevant questions here: Why were the medical examinations conducted in the interrogation room? Did the interrogators call the doctor because they were worried about the health of FT as a result of the application of "special means"? Or was the doctor present during the interrogations as indicated by FT?

In other words: Was the doctor complicit in application of "special means" / illtreatment or torture while monitoring the health of FT and guiding interrogators in preventing mishaps?

Apparently the inspector posed no such questions.

The suspicion that the doctor transgressed medical ethics ${ }^{2}$ is reinforced by

2 According to the UN standard for medical ethics in prisons:

It is a gross contravention of medical ethics, as well as an offence under applicable international instruments, for health personnel, particularly physicians, to engage, actively or passively, in acts which constitute participation in, complicity in, incitement to or attempts to commit torture or other cruel, inhuman or degrading treatment or punishment.

According to the WMA Tokyo Declaration: 1. The physician shall not countenance, condone or participate in the practice of torture or other forms of cruel, inhuman or degrading procedures (...) in all situations, including armed conflict and civil strife.

4..... physicians have the ethical obligation to report abuses...

6. The physician shall not be present during any procedure during which torture or any other forms of cruel, inhuman or degrading treatment is used or threatened. 
the gross insufficiency of the medical reports that could be taken as a cover-up, rather than reflecting mere incompetency.

It appears that both the UN and the World Medical Association (WMA) standards were violated by the doctor. The ruling did not at all treat the complaint about medical complicity in torture and ill-treatment.

Israel's medical association (IMA) member of the WMA - should have regulations and guidance to protect doctors working in places of detention from situations where they may violate provisions of the international standards and IMA ought to look into cases where information indicates that violations have taken place. IMA should work for the introduction in Israel of the Istanbul Protocol. It should advocate for the appointment or establishment of an independent institution where such examinations can take place and should facilitate trainings of doctors and psychologists in its use and trainings for legal professionals in the implications of the IP examination results. This is in line with the UN Committee against Torture's recommendations to Israel (2016) and the IMA would be a natural partner in the process.

\section{Non-institutional medical and psychological examinations of FT}

FT was examined by a medical doctor some five months after the interrogations applying "special means". A medical record is not reproduced in the ruling. It is criticized for lacking description of medical findings and diagnosis and that the opinion-that FT's symptoms from the eye and leg match his story-relies primarily on FT's complaints (para 55).

First, while it is correct that lack of diagnosis or assessment of the origin of symptoms and signs is a problem in medicolegal documents, a reader of the ruling could wonder why the same criticism was not applied against the documents written in the prison. Second, when a medico-legal examination is done it will have to rely on information from the person /patient and other available medical evidence. Apart from the low quality documents quoted above, sufficient medical evidence was not produced in a timely fashion by the authorities, the only actors who were empowered to do so.

FT was further thoroughly examined in December 2017 by a clinical psychologist and a psychiatrist internationally recognized for his extensive experience with the IP: Their report was not "granted real weight," because

a. it was only made available for review by the Inspector shortly before the court hearing.

b. It was prepared more than five years after the interrogation.

c. It was almost entirely based on FT's version (para 56).

\section{Cs. a. If information important for} establishing facts emerges it would be in the interest of the court to include it.

Cs. b. IP examinations may be carried out even after a long delay, but particularly physical marks after torture will disappear with time. Psychological symptoms may be long-lasting, even lifelong, e.g. the PTSD, (cf. Pérez-Sales, 2017). It is interesting that $51 / 4$ years delay is determined to be invalidating for the value of the report, while the value of the interviews by the inspector with the prison staff, some of which were conducted $3 \frac{1}{2}$ years after the event, 
was not questioned. Where is the time limit for acceptability?

Cs. c. The medical-psychological IP examination of a person who alleges exposure to torture is the UN standard for an assessment of the credibility /consistency of a statement about torture.

It is a very meticulous interview where specialists most often spend 5-10 hours with the examinee, sufficient to approach the history of torture /ill-treatment and symptoms from various angles, to assess the examinee's psychological and physical reactions to the examination and to assess the level of consistency between the elements of the examination, i.e. the credibility of the allegations.

It is not a police investigation and including witnesses' statements in the medical examination is rarely relevant, and then only to clarify health related issues, i.e. ailments and sequelae in the period after interrogation, detention and imprisonment. The medical examiners will not be mandated, empowered nor qualified to interview appropriately, e.g. police investigators as to their participation in a possible crime.

\section{The interests of the officers and the doctor}

An officer who has participated in torture or other potentially prohibited actions during interrogations will not have the slightest interest in shedding light on facts so as to avoid sanctions and to avoid implicating colleagues, which could make him a pariah in his institution. This lack of motivation would include record keeping that may not always be complete, particularly as to information about "special means".

Is it at all thinkable that interrogators who transgress the limit for the permissible would put that on record? Nevertheless, the logic of the Inspector seems to be that when something is not on record it did not happen. There are serious incentives for not putting all parts of the acts committed or medical evidence on record. Nevertheless, the Inspector and the judges regarded the records and the testimonies of officers and the doctor as the final truth.

\section{The ruling and the CAT}

As party to the CAT Israel has the obligation to perform prompt, and impartial examinations of complaints of torture (CAT $\$ \int 12,13$ ). The investigation by the authorities was far from prompt and the medical aspects far from sufficient; it was close to being non-existent and the sparse information quoted in the ruling was of very poor quality.

The UN Committee against Torture (2016) has recommended that "[Israel] urgently take the measures necessary to guarantee in practice that physicians and other medical staff dealing with persons deprived of liberty duly document all signs and allegations of torture or ill-treatment and report them without delay to the appropriate authorities."

The authorities should have referred FT for a full IP medical-psychological examination at the time when the first torture complaint was lodged. Such examinations could further have clarified FT's right to compensation, (cf. CAT $\ 14$ ). Moreover, FT should have been referred to a dentist and an ophthalmologist.

The issue of compensation for exposure to non-permitted means is not dealt with directly. However, the mild sentence (as quoted above) could be seen as the judge apparently recognizing that the interrogation procedures were unfair or reprehensible and leniency was used as a form of compensation without admitting openly 
what had happened to FT.

It appears that the establishment of FT's actions as terrorist activities is used as a justification for the means used against him, which violates the CAT $\ 2.2$.

The ruling does not mention Other Cruel, Inhuman and Degrading Treatment or Punishment ["ill-treatment"] in spite of the fact that ill-treatment is prohibited in the CAT. Since the "special means" used in the FT case is classified material it is impossible to see how the court distinguishes between "special means", (in principle unlawful, but subject to impunity at the discretion of the Attorney General) and ill-treatment / torture, which is prohibited. The lack of transparency in the administration of "special means" and the system of administrative impunity violates the obligation under the CAT for states to prevent torture and illtreatment $(\Omega 2.1$ and 16.1$)$. The points (a$\mathrm{h}$ ) in the following section can be seen as an illustration of a lack of will to prevent torture and ill-treatment.

\section{The ruling and its questionable foundation}

The court ruled that FT's claims were thoroughly and carefully investigated and that FT was not tortured (para 67) and that "there were no support for the claim that he had lost consciousness or that he suffered any physiological or psychological harm as a result of his arrest or interrogation (para 19).” The conclusion, inter alia, builds on

a. The very poor quality medical documentation from examinations in the period of "special means" usage.

b. Disregard of medical findings described in a).

c. Failure to conduct appropriate medicalpsychological examinations immediately after allegations of torture and illtreatment. d. The absence of consideration in the ruling of an IP examination made by an international and a local expert.

e. Misinterpretation of the concept of torture. One individual element (sleep deprivation) of the allegations was assessed as not constituting torture (para 51). Instead, the whole of the interrogating environment should have been assessed taking into consideration that the individual methods may not always amount to torture, but the application of them simultaneously will most likely constitute torture. Likewise, an individual symptom (vomiting) was assessed as not being a proof of torture (para 52). Instead, the entirety of FT's physical and psychological health should have been assessed through a full examination in accordance with the IP.

f. Institutional records (classified) and statements from interrogating officers and prison guards collected some $3 \frac{1}{2}$ years after the events were valued as the truth in spite of the obvious interest that officers have in not shedding light on potentially punishable actions, de facto approved or not approved beforehand by a superior.

g. Labeling FT as untrustworthy was, inter alia, based on FT's unwillingness to have a polygraph examination, the results of which are controversial. Polygraph tests, like any imperfect diagnostic tests, yield both false positive and false negative results and test performance is far below perfection and highly variable across situations ((National Research Council 2003, p.106).

h. The untrustworthiness of FT was further based on inconsistencies in his accounts over time. It should be considered that torture is designed to impair the cognitive functions of the victim through 
the application simultaneously of e.g. sleep deprivation, lengthy interrogations, physical exhaustion procedures and psychological pressure. Details may have been misperceived and on later occasions interpreted or just worded in different manners. Impaired memory and ability to concentrate are common symptoms experienced by victims of torture. It is not to be expected that a torture victim can recall all details in the same wording. The strength of the IP is that such differences are explored and assessed while taking the necessary time approaching such issues from various angles.

\section{Conclusion}

1. It is not for the documenting medical experts (Shir, 2019), but for the court to decide whether the level of pain and suffering inflicted reaches the threshold of torture [while disregarding ill-treatment], i.e., the court upholds the prerogative to apply its own interpretation of the definition of torture, despite existing medical evidence and disregarding the Istanbul Protocol. The criteria used to determine the level of FT's pain and suffering does not appear in the ruling.

2. The ruling states that the burden of proof-that the "means" were not reasonable [constituting torture]-falls upon the petitioner (para 36). In the light of the above (1, a-h) this is in practice impossible for the petitioner to establish. This aligns with Shir's statement that no ISA interrogator has been indicted in 1200 torture complaints.

\section{References}

National Research Council of the National Academies (2003). The Polygraph and Lie Detection. Washington, D.C. doi: https:/doi. org/10.17226/10420
Pérez-Sales, P (2017). Psychological Torture. Abingdon, UK and New York, USA. Routledge.

Shir E. (2019). How to say IP in Hebrew. The case of Mr. Firas Tbeish. Torture fournal 29(2), 90-95. https://doi.org/10.7146/torture.v29i2.114967

Firas Tbeish et al. v the Attorney General et al., HCJ 9018/17 (2018), retrieved from http://stoptorture. org.i1/wp-content/uploads/2017/02/F.-TbeishRuling-Nov. 2018.ENG_.pdf

United Nations Committee against Torture (2016). Concluding observations on the fifth periodic report of Israel. Retrieved from https://tbinternet. ohchr.org/_layouts/15/treatybodyexternal/ Download.aspx?symbolno=CAT/C/ISR $/$ $\mathrm{CO} / 5$ \&Lang=En

Convention against Torture and Other Cruel, Inhuman or Degrading Treatment or Punishment, New York 10 December 1984, United Nations Treaty Series, vol. 1465, 85. Retrieved from www.ohchr.org/ EN/ProfessionalInterest/Pages/CAT.aspx

UN Office of the High Commissioner for Human Rights (OHCHR), Manual on the Effective Investigation and Documentation of Torture and Other Cruel, Inhuman or Degrading Treatment or Punishment ("Istanbul Protocol”), 2004, HR/P/ PT/8/Rev.1. Retrieved from https://www.ohchr. org/_layouts/15/WopiFrame.aspx?sourcedoc $=\% 2$ FDocuments\%2FPublications\%2Ftraining8Rev1 en $\% 2$ Epdf\&action=view

UN General Assembly, Principles of Medical Ethics relevant to the Role of Health Personnel, particularly Physicians, in the Protection of Prisoners and Detainees against Torture and Other Cruel, Inhuman or Degrading Treatment or Punishment, 16 December 1983, A/RES/37/194. Retrieved from https://www.ohchr.org/EN/ProfessionalInterest/ Pages/MedicalEthics.aspx

UN General Assembly, United Nations Standard Minimum Rules for the Treatment of Prisoners (the Nelson Mandela Rules), 8 January 2016, A/ RES/70/175. Retrieved from https://www.ohchr. org/_layouts/15/WopiFrame.aspx?sourcedoc=/ Documents/ProfessionalInterest/ NelsonMandelaRules.pdf\&action $=$ default $\&$ Defa ultItemOpen $=1$

World Medical Association, Declaration of TokyoGuidelines for Physicians concerning Torture and Other Cruel, Inhuman or Degrading Treatment or Punishment in Relation to Detention and Imprisonment, October 1975. Retrieved from https://www.wma.net/policies-post/ wma-declaration-of-tokyo-guidelines-forphysicians-concerning-torture-and-other-cruelinhuman-or-degrading-treatment-or-punishmentin-relation-to-detention-and-imprisonment/ 